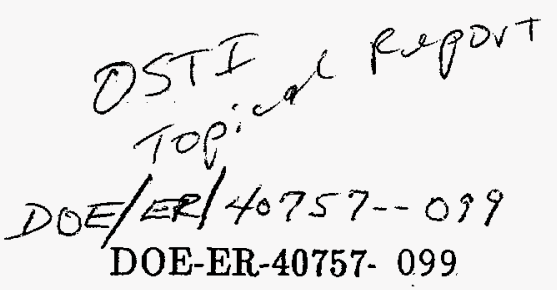

UTEXAS-HEP-97-11

MSUHEP-70610

\title{
Higgs-photon associated production at hadron colliders
}

\author{
Ali Abbasabadi ${ }^{1}$, David Bowser-Chao ${ }^{2}$, Duane A. Dicus ${ }^{3}$ and Wayne W. Repko ${ }^{4}$ \\ ${ }^{1}$ Department of Physical Sciences, Ferris State University, Big Rapids, Michigan 49307 \\ ${ }^{2}$ Department of Physics, University of Mlinois at Chicago, Chicago, Mlinois 60607 \\ ${ }^{3}$ Center for Particle Physics and Department of Physics University of Texas, Austin, Texas 78712 \\ ${ }^{4}$ Department of Physics and Astronomy Michigan State University, East Lansing, Michigan 48824
}

(June 17, 1997)

\begin{abstract}
We present cross sections for the reactions $p \bar{p} \rightarrow H \gamma$ and $p p \rightarrow H \gamma$ arising from the subprocess $q \bar{q} \rightarrow H \gamma$. The calculation includes the complete oneloop contribution from all light quarks and is the main source of Higgs-photon associated production in hadron colliders. At Tevatron energies, the cross section is typically $0.1 \mathrm{fb}$ or less, while at LHC energies it can exceed $1.0 \mathrm{fb}$.

14.80.Bn, 13.85.-t, 12.15.Ji

\section{DISCLAIMER}

This report was prepared as an account of work sponsored by an agency of the United States Government. Neither the United States Government nor any agency thereof, nor any of their employees, makes any warranty, express or implied, or assumes any legal liability or responsibility for the accuracy, completeness, or usefulness of any information, apparatus, product, or process disclosed, or represents that its use would not infringe privately owned rights. Reference herein to any specific commercial product, process, or service by trade name, trademark, manufacturer, or otherwise does not necessarily constitute or imply its endorsement, recommendation, or favoring by the United States Government or any agency thereof. The views and opinions of authors expressed herein do not necessarily state or reflect those of the United States Government or any agency thereof.
\end{abstract}

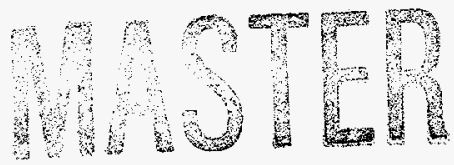




\section{DISCLAMMIXR}

Portions of this document may be illegible in electronic image products. Images are produced from the best available original document. 


\section{INTRODUCTION}

The production of intermediate mass Higgs bosons at hadron colliders arises primarily from the gluon fusion process $g g \rightarrow H$, which is dominated by the top quark loop [1]. Depending on the collider energy, there can also be a substantial contribution from the gauge boson fusion processes $W^{+} W^{-}, Z Z \rightarrow H$ [2]. For Higgs boson masses $m_{H}$ less than $2 m_{W}$, detection of the Higgs via its dominant decay mode $H \rightarrow b \bar{b}$ must contend with a large background from $g g \rightarrow b \bar{b}$. The production of Higgs bosons in association with a gauge boson, specifically $Z, W^{ \pm}[3,4]$ or a photon $[5,6]$, is potentially helpful in dealing with these backgrounds, although at a significant cost in the rate.

In this report, we examine the possibility of using Higgs-photon associated production in hadron collisions as a means of studying properties of the Higgs boson. The main source of the $H \gamma$ final state is quark-antiquark annihilation. Two gluon annihilation, which accounts for single $H$ production, is forbidden by Furry's theorem because the gluons are in a color singlet state. For light quarks, the direct annihilation into $\mathrm{H \gamma}$ is supressed by the ratio $m_{q} / m_{W}$ and is negligible. This means that one-loop electroweak corrections involving $W$ 's, $Z$ 's and top quarks dominate $q \bar{q} \rightarrow H \gamma$. The diagrams involved in the quark-antiquark annihilation calculation are similar to those encountered in the calculation of $e \bar{e} \rightarrow H \gamma$, which has been performed for the Standard Model [5,6] and its supersymmetric generalization [7]. In the next section, we outline the extension of our previous results [6] to $q \bar{q} \rightarrow H \gamma$. This is followed by a discussion.

\section{OUTLINE OF THE CALCULATION}

The amplitude for $q \bar{q} \rightarrow H \gamma$ receives contributions from pole diagrams involving virtual photon and $Z$ exchange and from various box diagrams containing quarks, gauge bosons and/or Goldstone bosons. There are also double pole diagrams whose contribution vanishes. This is illustrated in Fig. 1. The main difference between $e \bar{e}$ annihilation and $q \bar{q}$ annihilation occurs in the crossed box diagram of Fig. 1c. Since both members of a quark doublet are charged, there are additional crossed box diagrams containing $W$ 's. This contribution can be obtained from our $Z$ box results by merely changing the coupling and replacing $m_{Z}$ by $m_{W}$.

In the non-linear gauges we chose [6], the full amplitude consists of four separately gauge invariant terms: a photon pole, a $Z$ pole, $Z$ boxes and $W$ boxes. These amplitudes can be written as

$$
\begin{aligned}
\mathcal{M}_{\mathrm{pole}}^{\gamma}= & \frac{\alpha^{2} m_{W}}{\sin \theta_{W}} \bar{v}\left(p_{2}\right) \gamma_{\mu} u\left(p_{1}\right)\left(\frac{\delta_{\mu \nu} k \cdot\left(p_{1}+p_{2}\right)-k_{\mu}\left(p_{1}+p_{2}\right)_{\nu}}{s}\right) \hat{\epsilon}_{\nu}(k) \mathcal{A}_{\gamma}(s), \\
\mathcal{M}_{\mathrm{pole}}^{Z}= & \frac{\alpha^{2} m_{W}}{\sin ^{3} \theta_{W}} \bar{v}\left(p_{2}\right) \gamma_{\mu}\left(v_{q}+\gamma_{5}\right) u\left(p_{2}\right)\left(\frac{\delta_{\mu \nu} k \cdot\left(p_{1}+p_{2}\right)-k_{\mu}\left(p_{1}+p_{2}\right)_{\nu}}{\left(s-m_{Z}^{2}\right)+i m_{Z} \Gamma_{Z}}\right) \hat{\epsilon}_{\nu}(k) \mathcal{A}_{Z}(s), \\
\mathcal{M}_{\mathrm{box}}^{Z}= & -\frac{\alpha^{2} m_{Z}}{4 \sin ^{3} \theta_{W} \cos ^{3} \theta_{W}} \bar{v}\left(p_{2}\right) \gamma_{\mu}\left(v_{q}+\gamma_{5}\right)^{2} u\left(p_{1}\right)\left\{\left[\delta_{\mu \nu} k \cdot p_{1}-k_{\mu}\left(p_{1}\right)_{\nu}\right] \mathcal{B}_{Z}(s, t, u)\right. \\
& \left.+\left[\delta_{\mu \nu} k \cdot p_{2}-k_{\mu}\left(p_{2}\right)_{\nu}\right] \mathcal{B}_{Z}(s, u, t)\right\} \hat{\epsilon}_{\nu}(k)
\end{aligned}
$$




$$
\begin{aligned}
\mathcal{M}_{\text {box }}^{W}= & \frac{\alpha^{2} m_{W}}{2 \sin ^{3} \theta_{W}} \bar{v}\left(p_{2}\right) \gamma_{\mu}\left(1+\gamma_{5}\right)^{2} u\left(p_{1}\right)\left\{\left[\delta_{\mu \nu} k \cdot p_{1}-k_{\mu}\left(p_{1}\right)_{\nu}\right] \mathcal{B}_{W}(s, t, u)\right. \\
& \left.+\left[\delta_{\mu \nu} k \cdot p_{2}-k_{\mu}\left(p_{2}\right)_{\nu}\right] \mathcal{B}_{W}(s, u, t)\right\} \hat{\epsilon}_{\nu}(k),
\end{aligned}
$$

where $s=-\left(p_{1}+p_{2}\right)^{2}, t=-\left(k+p_{1}\right)^{2}$ and $u=-\left(k+p_{2}\right)^{2}$. Here, $v_{q}$ denotes the $q \bar{q} Z$ vector coupling constant, $v_{q}=1-4\left|e_{q}\right| \sin ^{2} \theta_{W}$, and $e_{q}$ is the quark charge in units of the proton charge. In terms of the scalar functions defined in the appendices of our previous paper [6], we have [8]

$$
\begin{aligned}
\mathcal{A}_{\gamma}(s)= & -e_{q}\left\{4\left(6+\frac{m_{H}^{2}}{m_{W}^{2}}\right) C_{23}\left(s, m_{H}^{2}, m_{W}^{2}\right)-16 C_{0}\left(s, m_{H}^{2}, m_{W}^{2}\right)\right. \\
& \left.-\frac{16}{3} \frac{m_{t}^{2}}{m_{W}^{2}}\left(4 C_{23}\left(s, m_{H}^{2}, m_{t}^{2}\right)-C_{0}\left(s, m_{H}^{2}, m_{t}^{2}\right)\right)\right\} \\
\mathcal{A}_{Z}(s)= & -2 I_{3}\left\{\left(5-\tan ^{2} \theta_{W}+\frac{m_{H}^{2}}{2 m_{W}^{2}}\left(1-\tan ^{2} \theta_{W}\right)\right) C_{23}\left(s, m_{H}^{2}, m_{W}^{2}\right)\right. \\
& +\left(\tan ^{2} \theta_{W}-3\right) C_{0}\left(s, m_{H}^{2}, m_{W}^{2}\right) \\
& \left.-\frac{1}{2} \frac{m_{t}^{2}}{m_{W}^{2}} \frac{1-(8 / 3) \sin ^{2} \theta_{W}}{\cos ^{2} \theta_{W}}\left(4 C_{23}\left(s, m_{H}^{2}, m_{t}^{2}\right)-C_{0}\left(s, m_{H}^{2}, m_{t}^{2}\right)\right)\right\}, \\
\mathcal{B}_{Z}(s, t, u)= & -\tilde{e}_{q} A(s, t, u), \\
\mathcal{B}_{W}(s, t, u)= & -2 I_{3}\left[A_{1}(s, t, u)+A_{2}(s, u, t)\right]+\tilde{e}_{q} A^{\prime}(s, t, u),
\end{aligned}
$$

with $I_{3}$ denoting the third component of the external quark weak isospin, $m_{t}$ being the top quark mass and the prime denoting the replacement $m_{Z} \rightarrow m_{W} . \tilde{e}_{q}$ is the charge of the internal quark in units of the proton charge.

The differential cross section $d \sigma(q \bar{q} \rightarrow H \gamma) / d \Omega_{\gamma}$ is

$$
\frac{d \sigma(q \bar{q} \rightarrow H \gamma)}{d \Omega_{\gamma}}=\frac{1}{256 \pi^{2}} \frac{s-m_{H}^{2}}{s^{2}} \sum_{\text {spin }}|\mathcal{M}|^{2},
$$

where the invariant amplitude $\mathcal{M}$ is the sum of Eqs. (1-4). To obtain the collider cross sections, we convolute Eq. (9) with the appropriate quark and antiquark structure functions using

$$
\frac{d \sigma}{d m_{H \gamma}^{2}}=\frac{1}{s} \int_{\tau}^{1} \frac{d x}{x} f_{q}(x) f_{\bar{q}}(\tau / x) \int_{-z_{0}}^{z_{0}} d z \frac{d \sigma(q \bar{q} \rightarrow H \gamma)}{d z} .
$$

Here, $\tau=m_{H \gamma}^{2} / s, z=\cos \theta_{\gamma}$ and $z_{0}=\cos \theta_{\min }$ is determined by the choice of the rapidity cut. We used CTEQ-1M structure functions [9] and a rapidity cut of 2.5 on both the Higgs boson and the photon.

\section{DISCUSSION}

The total cross section for $p \bar{p}(p p) \rightarrow H \gamma$ is illustrated in Fig. 2 for both Tevatron and $\mathrm{LHC}$ energies. At the Tevatron, it is clear that an upgrade in luminosity to $20-30 \mathrm{fb}^{-1} /$ year 
is necessary for $H_{\gamma}$ to be seen. Luminosities of this order are contemplated in the TeV33 working group study [10]. For the LHC with a proposed luminosity of $100 \mathrm{fb}^{-1} /$ year, associated production for $m_{H}<200 \mathrm{GeV}$ will be observable.

In addition to $H \gamma$ production from light quark annihilation by means of one-loop amplitudes, there is the possibility of heavy quark annihilation at the tree level. As mentioned in the introduction, the tree level amplitudes are surpressed by a factor of $m_{q} / m_{W}$, which, while negligible for $u$ and $d$ quarks, could be significant for $s$ and $c$ quarks $[11,12]$. We specifically checked the contribution of the $s \bar{s}$ tree level amplitude and found that for $m_{H}<m_{Z}$, the one-loop $s \bar{s}$ amplitude dominates because of the $Z$ pole. When $m_{H}>m_{Z}$, the tree level contribution exceeds the one-loop contribution. However, in this case, the $s$ quark luminosity is so small that both contributions are negligible compared to that of the light quarks.

Finally, to determine the sensitivity of associated production to changes in the $t \bar{t} H$ coupling, we computed the cross section including a factor $\lambda$ multiplying the Standard Model coupling [13]. For a $2 \mathrm{TeV}$ Tevatron upgrade, this effect is illustrated in Fig. 3 for $0.0 \leq \lambda \leq 4.0$ as a function of $m_{H}$, and in Fig. 4 as a function of $\lambda$. The most obvious effect is the uniform decrease in the cross section as $\lambda$ varies from $\lambda=0$ to the Standard Model value at $\lambda=1.0$. With increasing $\lambda$, the cross sections eventually exceed the Standard Model result. For larger values of $m_{H}$, the observation of $H \gamma$ events at a $2 \mathrm{TeV}$ machine with a luminosity of $20-30 \mathrm{fb}^{-1} /$ year would necessitate a non Standard Model coupling.

\section{ACKNOWLEDGMENTS}

We would like to thank G. Kane for several helpful conversations. This research was supported in part by the U.S. Department of Energy under Contract No. DE-FG01393ER40757 and in part by the National Science Foundation under Grant No. PHY-93-07980. 


\section{REFERENCES}

[1] J. F. Gunion, H. E. Haber, G. Kane, and S. Dawson, The Higgs Hunter's Guide (Addison-Wesley, Menlo Park, CA, 1990).

[2] S. Petcov and D. R. T. Jones, Phys. Lett. B84, 440 (1979); R. N. Cahn and S. Dawson, Phys. Lett. B136, 196 (1984), B138, 464 (1984) (E); G. L. Kane, W. W. Repko and W. B. Rolnick, Phys. Lett. B148, 367 (1984); S. Dawson, Nucl. Phys. B 249, 42 (1985).

[3] J. D. Bjorken, in Proceeding of the SLAC Summer Institute on Particle Physics, Stanford, CA (1976); SLAC Pub 1866 (1977).

[4] S. Mrenna and G. L. Kane, Possible detection of a Higgs boson at higher luminosity hadron colliders, hep-ph/9406337 (1994) (unpublished).

[5] A. Barroso, J. Pulido and J. C. Romão, Nucl. Phys. B267, 509 (1986).

[6] A. Abbasabadi, D. Bowser-Chao, D. A. Dicus and W. W. Repko, Phys. Rev D 52, 3919 (1995).

[7] A. Djouadi, V. Driesen, W. Hollick and J. Rosiek, University of Karlsruhe preprint KA-TP-21-96, hep-ph/9609420.

[8] Equations (5) and (6) contain an additional factor of 2 in the top quark contribution compared to Ref. [6].

[9] CTEQ Collaboration: J. Botts, et al., Phys. Lett. B 304, 159 (1993); H. L. Lai, et al., Phys. Rev. D 51, 4763 (1995).

[10] P. P. Bagley, et al., Summary of the TEV3९ Working Group, FERMILAB-CONF 96392 , Oct. 1996.

[11] V. A. Litvin and F. F. Tikhonin, Associated production of $H \gamma$ and $H Z$ pairs at $\mu^{+} \mu^{-}$ collisions, hep-ph/9704417 (1997).

[12] A. Abbasabadi, D. Bowser-Chao, D. A. Dicus and W. W. Repko (in progress).

[13] H. E . Haber, G. L. Kane and T. Sterling, Nuc. Phys. B 161, 493 (1979). 


\section{FIGURES}

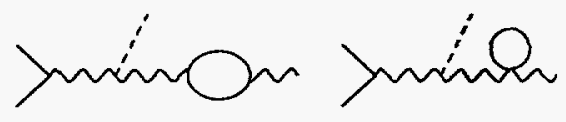

(a)

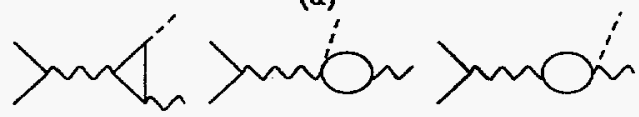

(b)
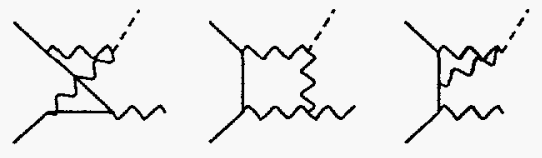

(c)

FIG. 1. Typical diagrams for the double pole (a), single pole (b) and box (c) corrections are shown. An external solid line represents a quark, a wavy line a gauge boson, a dashed line a Higgs boson and an internal solid line a quark, gauge boson, Goldsone boson or ghost.

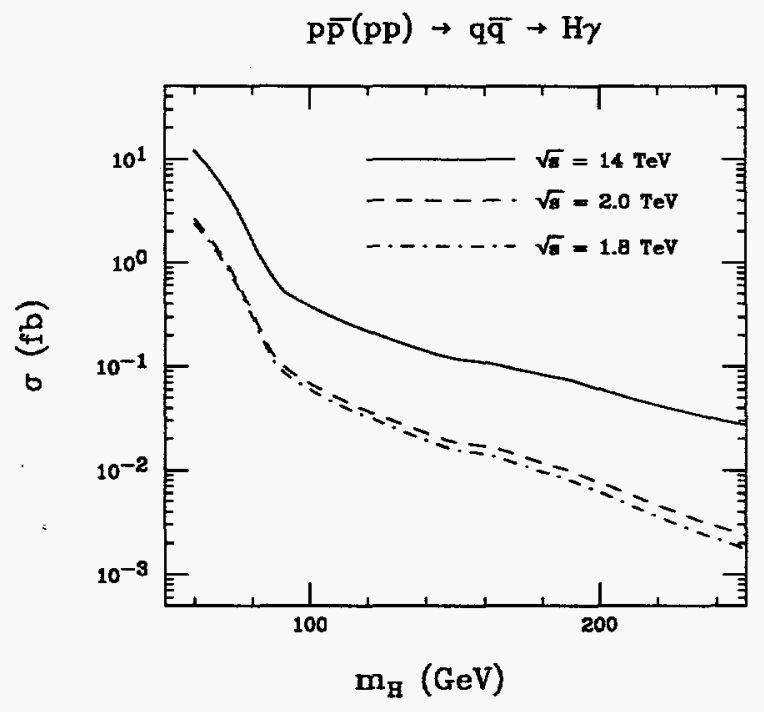

FIG. 2. The total cross section for $H_{\gamma}$ production from $q \bar{q}$ annihilation in $p \bar{p}$ and $p p$ scattering is shown for various collider energies. The dot-dashed line corresponds to the current Tevatron energy, the dashed line to an upgraded Tevatron energy and the solid line to the proposed LHC energy. 


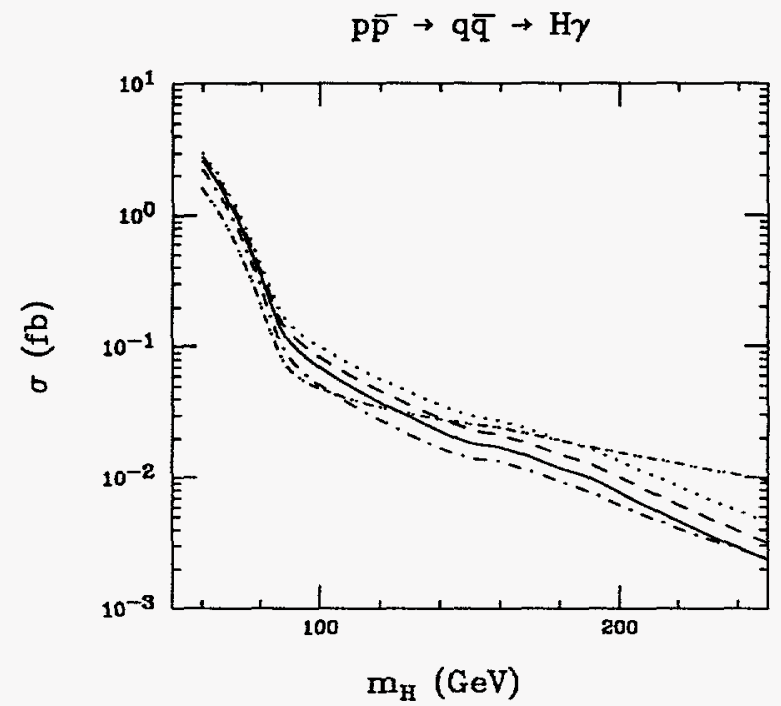

FIG. 3. The total cross section for $H \gamma$ production from $q \bar{q}$ annihilation in $p \bar{p}$ scattering is shown for varying strengths of the $t \bar{t} H$ coupling (in multiples $\lambda$ of the Standard Model coupling) at an upgraded Tevatron. The solid line is $\lambda=1$, the dot-dashed line $\lambda=2$, the dot-dot-dashed line $\lambda=4$, the dashed line $\lambda=.5$, and the dotted line $\lambda=0$.

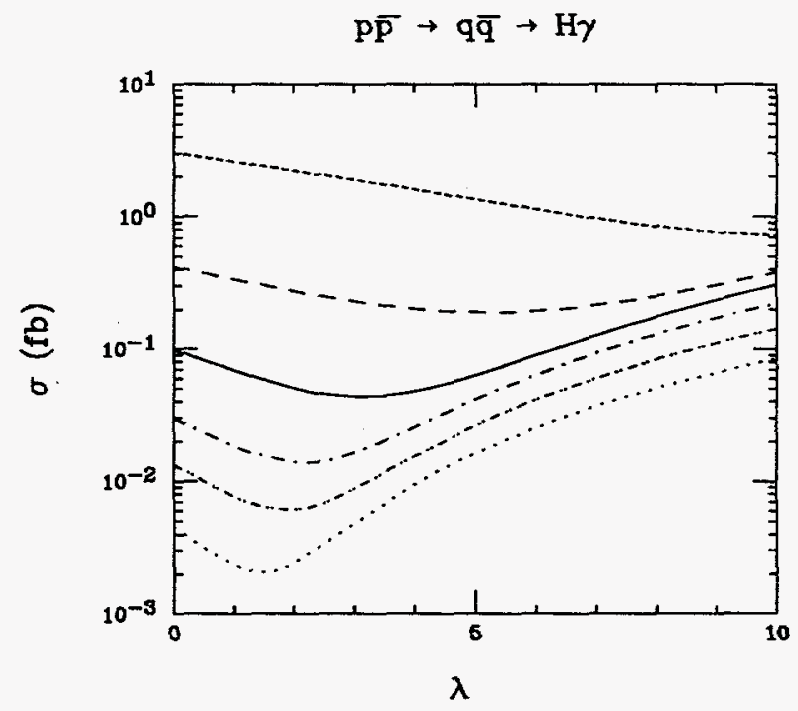

FIG. 4. The total cross section for $H \gamma$ production from $q \bar{q}$ annihilation in $p \bar{p}$ scattering is shown as a function of the $t \bar{t} H$ coupling (in multiples $\lambda$ of the Standard Model coupling) at an upgraded Tevatron for several values of $m_{H}$. The short dashed line is $m_{H}=60 \mathrm{GeV}$, the dashed line $m_{H}=80 \mathrm{GeV}$, the solid line $m_{H}=100 \mathrm{GeV}$, the dot-dased line $m_{H}=150 \mathrm{GeV}$, the dot-dot-dashed line $m_{H}=200 \mathrm{GeV}$ and the dotted line $m_{H}=250 \mathrm{GeV}$. 